\title{
La transvaloración de las perspectivas. Nietzsche y la crítica de la cultura desde el punto de vista del valor*
}

\author{
The transvaluation of perspectives. Nietzsche and the critique \\ of culture from the point of view of value
}

MARINA GARCÍA-GRANERO**

\begin{abstract}
Resumen: El artículo analiza el estrecho vínculo entre perspectivismo, genealogía y fisiología en la filosofía de Nietzsche. La tesis cardinal es que el punto de vista del valor es más fundamental que el de la verdad. El método genealógico posibilita el estudio de las condiciones de conservación y crecimiento de los puntos de vista y los valores, siguiendo el hilo conductor del cuerpo, con el objetivo de crear nuevos resortes de sentido y salir del nihilismo. Se presta especial atención a la tensión entre dogmatismo y relativismo. Finalmente expongo la noción de "cría" (Züchtung), que posibilita un cuestionamiento radical de la cultura y pone de relieve el carácter fisiológico de la moral.

Palabras clave: perspectivismo, valor, cría, moral, punto de vista, interpretación.
\end{abstract}

\begin{abstract}
The article analyses the close link between perspectivism, genealogy and physiology in Nietzsche's philosophy. The cardinal thesis is that the point of view of value is more fundamental than that of truth. The genealogical method enables the study of the conditions of conservation and growth of points of view and values, following the conductive thread of the body, with the aim of creating new springs of meaning and leaving nihilism behind. Special attention is paid to the tension between dogmatism and relativism. Lastly, I present the notion of "breeding" (Züchtung), which enables a radical questioning of culture and highlights the physiological character of morals.

Keywords: perspectivism, value, breeding, moral, point of view, interpretation.
\end{abstract}

\section{Introducción}

Contra el nihilismo de una humanidad cansada, agotada, llena de dudas sobre sí misma y sobre su propia historia, Nietzsche decide realizar una crítica liberadora de la cultura desde la óptica de la vida. El perspectivismo permite elaborar otras interpretaciones con poder para cambiar los resortes del sentido, para dejar de vivir de un modo en el que deja de tener

Recibido: 13/06/2018. Aceptado: 21/10/2018.

* Este artículo se enmarca dentro del proyecto de investigación FFI2016-76753-C2-1-P, financiado por el Ministerio de Economía y Competitividad.

** Investigadora FPU-MCIU en el Departamento de Filosofía de la Universitat de València (Referencia de la ayuda: FPU15/04085). Entre sus publicaciones sobre la filosofía de Nietzsche cabe destacar "Nietzsche y el mejoramiento humano. Reflexiones en torno a la noción de vida" (Isegoría. Revista de Filosofía Moral y Política, $\mathrm{n}^{\circ} 57,2017$, pp. 599-615). Contacto: marina.garcia-granero@uv.es. 
sentido vivir ${ }^{1}$. No busca una multiplicación de las interpretaciones en tanto que aumento del saber en sí mismo, sino que pretende alcanzar las raíces reflexivas del horizonte de sentido del conjunto de la cultura moderna, y desde ahí llevar a cabo la transvaloración de los valores.

Sin duda el perspectivismo es un aspecto del pensamiento de Nietzsche que ha levantado mucho interés y que cuenta con una tradición de estudios entre los intérpretes de su obra. A día de hoy, se siguen desarrollando interesantes discusiones epistémicas en torno a la perspectiva del perspectivismo, el valor de la falsedad, y si acaso la tesis del perspectivismo se puede aplicar a todo tipo de conocimiento. No obstante, el presente artículo se propone incidir en la peculiar crítica de la cultura y de los valores que resulta del perspectivismo, desde la filosofía moral, política y social.

El problema de la verdad y de la perspectiva en la filosofía de Nietzsche no se reduce a la cuestión de la representación o proyecto de demostración lógica. Tampoco puede ser entendido meramente en términos epistémicos, sino que sirve como preámbulo de la pregunta por el valor de los valores, y constituye una nueva crítica que desenmascara las formaciones de poder y los constructos ideológicos que fundamentan la dominación del ser humano, entendiendo la moral como un código histórico de poderes en lucha. De modo que, en el artículo, se estudia el perspectivismo como método propiamente nietzscheano de interpretación de los fenómenos morales, tal y como es presentado en obras como Más allá del bien y el mal, La genealogía de la moral y El crepúsculo de los ídolos.

En primer lugar, analizaremos la introducción del perspectivismo en los escritos de Nietzsche y sus consecuencias para la comprensión del valor en la filosofía moral. En segundo lugar, se analizará el método de la genealogía poniendo de relieve su pretensión de crear conocimiento útil y válido que siente los cimientos para la posterior transvaloración. Finalmente, se estudiará el concepto de "cría", como perspectiva que aspira a crear una reconciliación entre natura y cultura y que pone de relieve los modos en que la moral deviene fisiología y cambia la vida humana.

\section{El perspectivismo y la comprensión del valor}

Como hemos adelantado en la introducción, en este trabajo daremos prioridad al perspectivismo moral. Vale la pena destacar que ya en Más allá del bien y del mal, Nietzsche expresa en una máxima la tesis del perspectivismo aplicado a la moral: «No existen fenómenos morales, sino sólo una interpretación moral de los fenómenos...» $\gg^{2}$. Dicho de otro modo, no existen hechos intrínsecamente morales, sólo interpretaciones morales, cuyo origen, a su vez, es extra-moral.

Aunque los términos "perspectiva", "perspectivista", o incluso "perspectividad" (Perspektivität), aparecen con frecuencia desde sus primeros escritos ${ }^{3}$, Nietzsche emplea el sustantivo "perspectivismo" (Perspektivismus) por primera vez en dos fragmentos póstumos de

1 El Anticristo $\$ 43(=\mathrm{AC})$.

2 Más allá del bien y del mal $\$ 108$ (= MBM), cf. MBM $\$ 34$ y El crepúsculo de los ídolos (=CI) «Los "mejoradores" de la humanidad» $§ 1$.

3 Se pueden consultar los usos de un término en la obra de Nietzsche en la edición crítica digital (eKGWB): http://www.nietzschesource.org. 
final de 1886 y comienzo de 1887 . De estos dos fragmentos ${ }^{4}$, el que por su mayor longitud ha pasado a ser conocido es el segundo, que reproduciremos a continuación en su totalidad, y en el que Nietzsche emplea el término "perspectivismo" 5 para poner de relieve que todo conocimiento nace de una determinada apertura al mundo y un punto de vista:

Contra el positivismo, que se queda en el fenómeno «sólo hay hechos», yo diría no, precisamente no hay hechos, sólo interpretaciones. No podemos constatar ningún factum «en sí»: quizás sea un absurdo querer algo así. «Todo es subjetivo», decís vosotros, pero ya eso es interpretación, el «sujeto» no es algo dado sino algo inventado y añadido, algo puesto por detrás - ¿ ¿Es en última instancia necesario poner aún al intérprete detrás de la interpretación? Ya eso es invención, hipótesis.

En la medida en que la palabra «conocimiento» tiene sentido, el mundo es cognoscible: pero es interpretable de otro modo, no tiene un sentido detrás de sí, sino innumerables sentidos, «perspectivismo».

Son nuestras necesidades las que interpretan el mundo: nuestros impulsos y sus pros y sus contras. Cada impulso es una especie de ansia de dominio, cada uno tiene su perspectiva, que quisiera imponer como norma a todos los demás impulsos ${ }^{6}$.

Para Nietzsche, la pregunta por el conocimiento y su posibilidad sólo es posible si se sabe qué es conocimiento, o si se cree en el conocimiento, y ya eso es interpretación. El perspectivismo representa, primordialmente, una crítica del estatus epistémico privilegiado otorgado a conceptos como "cosa en sí", "verdad", "hecho", "realidad", y su carácter de "dado", producto de una perspectiva que ya está impregnada de estimaciones de valor, precisamente por su ser perspectiva. Ni el sentido ni el carácter moral de una acción pueden ser encontrados o descubiertos, sino que han de ser introducidos por el ser humano ${ }^{7}$.

Contra el intento positivista de enmascarar los intereses que mueven a todo sujeto, Nietzsche destaca el carácter necesariamente valorativo e interesado de todo conocimiento, pues también las ciencias naturales responden a un cúmulo de intereses, necesidades y condiciones de supervivencia del ser humano en tanto que especie. De modo que el error de los positivistas no es de contenido, sino metodológico, ya que niegan la naturaleza, estructura, función y modo de existencia peculiar de la perspectiva, que es condición básica de la vida. El propio conocimiento siempre está lejos de ser contemplación desinteresada y la ciencia también surge como voluntad de poder y dominio del mundo. La perspectiva en Nietzsche cumple una función afirmativa porque el punto de vista nutre la interpretación, forma parte

4 El primer fragmento se compone únicamente de una sentencia: «Perspectivismo de la deseabilidad (del ideal)» (FP 1886-1887 7[21], vol. IV p. 215). Al citar los Fragmentos Póstumos, indicaré en primer lugar el año y el número de fragmento, para después situarlos en la edición en español publicada por Tecnos en cuatro volúmenes.

5 Sobre las fuentes del neologismo «perspectivismo», cf. Gori, Pietro (2017), Nietzsche y el perspectivismo, Córdoba (Argentina): Editorial Brujas, pp. 88-93.

6 FP 1886-1887 7[60], vol. IV p. 222. El énfasis en los textos nietzscheanos sigue las convenciones académicas establecidas desde la edición de Colli y Montinari: las palabras en itálica son las que Nietzsche subrayó una vez en el manuscrito.

7 Gori, Pietro y Stellino, Paolo (2018), «Moral Relativism and Perspectival Values», en: António Marques and João Sàágua (eds.), Essays on Value and Practical Rationality, Bern: Peter Lang, pp. 155-174, p. 160. 
de ella y de nuestra experiencia del mundo. No es posible conocer lo que las cosas son en sí mismas, de una manera independiente a nuestra apropiación interpretativa: «No hay un «hecho» en sí, sino que siempre tiene que introducirse primero un sentido para que pueda haber un hecho (...) En la base está siempre «¿qué es eso para mí?» (para nosotros, para todo lo que vive, etc.)»8 .

Toda apertura al mundo y experiencia de la realidad está ligada a una perspectiva específica, a unos parámetros que posibilitan una particular experiencia del mundo y que hacen que ver sea ver-algo. Nuestro punto de vista orienta la mirada en una determinada dirección, perímetro y horizonte: «estamos en nuestra tela como la araña, y sólo podemos atrapar con ella justo aquello que se deja enredar en nuestra tela» ${ }^{9}$. El perspectivismo pretende situar los límites sobre lo que podemos saber y, como tal, constituye una doctrina de abasto mucho más epistemológico que ontológico. No versa sobre "lo que hay" sino, en primera instancia, sobre "cómo podemos conocer", y en segunda instancia, sobre el valor de dichos conocimientos. Las preguntas críticas desde la perspectiva de la vida ${ }^{10}$ no son ontológicas, no tratan de demostrar la existencia de un objeto, sino que tratan de elucidar por qué tal objeto debe ser, o no, importante para nosotros, o por qué un valor debe considerarse valioso.

Ahora bien, el perspectivismo no equivale ni a escepticismo ni a rechazo de las ciencias, sino que abre un espacio de transvaloración de los caracteres científicos y de crítica frente a sus pretensiones de acceso exclusivo al conocimiento. Nietzsche prestó gran atención al contexto científico de su época, y adopta una perspectiva que podríamos considerar "naturalizante"11. Le importaba crear interpretaciones que pudiesen ser acreditadas científicamente, sin por ello situar a las ciencias naturales por encima de la filosofía, ya que no hemos de olvidar la cuestión más difícil de todas: «saber si la ciencia está en disposición de proporcionar metas a la acción, después de haber demostrado que puede retirarlas y destruirlas» ${ }^{12}$. La ciencia no puede ser nuestra guía cuando se trata de tomar decisiones morales, ya que una perspectiva meramente naturalista no es capaz ni de crear valores, ni de elucidar el valor de los valores. «La naturaleza siempre carece de valor» ${ }^{13}$, y todo lo que tiene valor en el mundo, no lo posee en sí, sino sólo porque nosotros se lo hemos otorgado, hemos proyectado nuestra apreciación del valor (Wertschätzung) al mundo.

El enfoque nietzscheano de la ciencia ofrece una reflexión hermenéutica sobre su significado desde la perspectiva de la vida y sus fuerzas poéticas, para así reconectar los resortes del pensamiento, la creación y la vida. Nietzsche no fue un filósofo de la ciencia, sino un crítico de la cultura, entendida en un sentido integral, y es precisamente como parte de dicha crítica de la cultura donde plantea el problema de la ciencia ${ }^{14}$, la pregunta crítica por

8 FP 1885 2[149], vol. IV p. 123.

9 Aurora $\$ 117(=\mathrm{A})$

10 Sobre la noción de vida en Nietzsche, cf. García-Granero, Marina (2017). «Nietzsche y el mejoramiento humano. Reflexiones en torno a la noción de vida», Isegoría. Revista de Filosofía Moral y Política, $\mathrm{n}^{\circ}$ 57, pp. 599-615.

11 Heit, Helmut (2016), «Naturalizing Perspectives. On the Epistemology of Nietzsche's Experimental Naturalizations», Nietzsche-Studien, $\mathrm{n}^{\circ} 45$, pp. 56-80.

12 La gaya ciencia $\$ 7$ (= GC).

13 GC $\$ 301$

14 Cf. Babich, Babette E. (2008), «Un problema con cuernos... el problema de la ciencia misma. La crítica de Nietzsche a la razón científica». Estudios Nietzsche, no 8 , pp. 13-52. 
su sentido y su rendimiento para la vida. «El espíritu de la ciencia, poderoso en la parte, pero no en el todo» ${ }^{15}$ : la ciencia no puede responder a las preguntas del sentido, del hacia dónde y el para qué. Por ello, una cultura superior debe dotar al ser humano de un doble cerebro, para sentir la ciencia y también lo que no es ciencia, «es esta una exigencia de la salud» ${ }^{16}$. El perspectivismo nos permite ser capaces de elaborar otra interpretación moral de los fenómenos, desde otra actitud frente al mundo, «una nueva conciencia de sí mismo y de la propia libertad: una emancipación para elegir los propios puntos de vista» ${ }^{17}$. No es la verdad, sino el poder, lo que nos hace libres.

Ahora bien, la filosofía de la interpretación no debe derivar en relativismo. El núcleo fuerte del perspectivismo nietzscheano y de la genealogía reside en que la problemática del valor es mucho más fundamental que la de la verdad: «la cuestión de los valores es más fundamental que la cuestión de la certeza: la última sólo adquiere seriedad en el supuesto de que la cuestión del valor haya tenido respuesta» ${ }^{18}$. Los valores son creencias interiorizadas que traducen las preferencias fundamentales de un tipo humano, la manera en que ordena la realidad y selecciona lo que considera prioritario, necesario, beneficioso o perjudicial. $\mathrm{Y}$ aunque podamos elaborar una infinidad de interpretaciones a partir de un texto o puntos de vista sobre un suceso, no todos ellos valen. Hemos de cuestionar los pensamientos, las teorías y las opiniones en términos de valor, ya que se alcanza un análisis más radical.

Nietzsche define en una anotación póstuma lo que él entiende por el "nihilismo activo"19. Nihilista activa es aquella persona capaz de disolver convicciones y artículos de fe, de destruir ídolos, pero que aún no posee la fortaleza suficiente para crear su propia meta, para determinar su propio porqué. Porque nihilismo significa falta de meta ${ }^{20}$, y la tarea más ardua no es eliminar la creencia en Dios, sino llevar a cabo la misma creación de los valores, labor que hasta entonces era delegada a la moral cristiana. Tras la muerte de Dios, surge el riesgo de que otras instancias (como la ciencia, el Estado, el mercado, la patria o la raza) ocupen su puesto y se encarguen de responder a las preguntas sobre el sentido y los fines últimos. Por tanto, el nihilismo permanecerá hasta que asumamos la carga de crear valores desde nuestra propia autonomía y destruyamos el hábito de considerar como "dados" los fines de nuestras acciones y nuestra vida.

Desde la perspectiva de la praxis vital, una falsedad no supone ninguna objeción:

La falsedad de un juicio no es para nosotros ya una objeción contra él (...). La cuestión está en saber hasta qué punto ese juicio favorece la vida, conserva la vida, conserva la especie, quizás incluso selecciona la especie; y nosotros estamos inclinados por principio a afirmar que los juicios más falsos (de ellos forman parte los juicios sintéticos a priori) son los más imprescindibles para nosotros, que el ser humano no podría vivir si no admitiese las ficciones lógicas, si no midiese la realidad con el

\footnotetext{
15 Humano, demasiado humano $\S 6$ y $\S 7$ (= HDH)

$16 \mathrm{HDH} \S 251$

17 Conill, Jesús (2008), «El significado de la ciencia y su poetización desde Nietzsche», Estudios Nietzsche, $\mathrm{n}^{\circ} 8$, pp. 53-64, p. 60.

18 FP $18867[49]$, vol. IV p. 220.

19 FP 1887 9[35], vol. IV p. 241-242.

20 FP 1887 7[61], vol. IV p. 223.
} 
metro del mundo puramente inventado de lo incondicionado, idéntico-a-sí-mismo, si no falsease permanentemente el mundo mediante el número, - que renunciar a juicios falsos sería renunciar a la vida, negar la vida ${ }^{21}$.

El uso práctico de una perspectiva no sirve como criterio de verdad, pero sí como criterio de valor, porque los errores y las ilusiones son instrumentos de conservación de las formaciones sociales y las costumbres, que se convierten en condiciones de vida ${ }^{22}$. «Que la verdad sea más valiosa que la apariencia, eso no es más que un prejuicio moral» y «no existiría vida alguna a no ser sobre la base de apreciaciones y de apariencias perspectivistas» ${ }^{23}$. Nietzsche desarrolla la conciencia crítica de que el lenguaje no refleja la verdad, sino que configura una apariencia. La comprensión y el pensamiento humanos han de contar con esta mentira originaria, por ello, hemos de estimar también el no-saber, el ver en general y en conjunto, el simplificar y falsear. Así, en Nietzsche el papel del intelecto se convierte en un problema que ha de ser respondido de manera histórico-hermenéutica ${ }^{24}$. Una vez hemos renunciado al "en sî" y eliminado estos presupuestos de la razón positivista, nos abrimos a la creación de sentido por parte del ser humano.

\section{Genealogía, conocimiento y objetividad}

A continuación, entramos en la discusión acerca de la genealogía, sin duda un terreno muy transitado dentro de los estudios nietzscheanos, pero considero necesario su análisis para una mejor comprensión y contextualización de la tesis que defiendo sobre la primacía del punto de vista del valor, y para preparar la reflexión final sobre el concepto de "cría".

Nietzsche critica que el punto de vista de la conciencia ha sido el prevaleciente tanto en la ciencia como en la cultura, y se han relegado a un segundo plano las capacidades sensibles del cuerpo para crear sentido. Dicho estatus privilegiado de la conciencia tiene un sentido eminentemente instrumental en la medida en que «la conciencia no pertenece a la existencia individual del ser humano, sino más bien a lo que en él es de naturaleza comunitaria y gregaria» ${ }^{25}$. Esta situación ascética ha provocado el empequeñecimiento del ser humano y un desprecio del sí mismo corporal, cuando en realidad, el cuerpo es tanto el punto de partida (Ausgangspunkt) como el hilo conductor (Leitfaden) que hace posible la creación del conocimiento y de la valoración. El cuerpo realiza «una actividad intelectual que no entra en la conciencia», es «un acontecer interno, por tanto, un asimilar, expeler, crecer, etc...» ${ }^{26}$. Así pues, la representación correcta de la índole de nuestra subjetividad es corporal: «cuerpo (Leib) soy yo íntegramente, y ninguna otra cosa», «instrumento de tu cuerpo es también tu pequeña razón, a la que llamas "espíritu"» ${ }^{27}$.

21 MBM §4. Cf. FP 1885 35[37], vol. III p. 781.

22 GC $\$ 121$.

23 MBM $\$ 34$

24 Barrios, Manuel (1999), «Intelecto logificador y voluntad creadora en Friedrich Nietzsche», Daimon, $\mathrm{n}^{\circ}$ 18, pp. 99-111.

25 GC $\$ 354$.

26 FP 1885 40[15], vol. III p. 848.

27 Así hablo Zaratustra, I «De los despreciadores del cuerpo» (= Za). 
Así pues, Nietzsche reconoce que, debido al punto de partida que representa nuestro cuerpo particular, nuestro conocimiento es limitado, lo cual no conlleva que el cuerpo sea un obstáculo al conocimiento. Contra toda la tradición platónica que entiende la ruptura con el cuerpo como condición de conocimiento auténtico y que deseca los conceptos convirtiéndolos en «momias conceptuales» ${ }^{28}$, Nietzsche defiende el cuerpo como punto de partida del conocimiento: vivir es condición del conocimiento y el conocimiento del que somos capaces es alcanzado gracias al cuerpo. Ahora bien, dicho conocimiento no es absoluto ni existe en sí mismo, sino que es perspectivista y limitado. No hay ninguna escapatoria, ningún otro camino desviado que nos permita adentrarnos en un supuesto mundo "real"; no existe una realidad no-perspectivista que pueda ser revelada por vía "racional". Es en la corporalidad donde el auténtico instinto de vida coloca la verdad de la manera más incondicional ${ }^{29}$, es la instancia con más potencial de probidad desde el punto de vista de la vida.

El método de la "genealogía" - término aparece por primera vez en el propio título de La genealogía de la moral (1887) - permite elaborar una explicación fisiológica de la moral $^{30}$. Se trata de una investigación regresiva que busca elucidar la proveniencia de los instintos y las fuentes productoras de un valor o de una interpretación, ya sea religiosa, política, etc. La problematización de dichas fuentes, de las condiciones y las circunstancias en que han emergido dichos valores y del recorrido que han atravesado hasta el presente, es el paso previo a su transvaloración. La genealogía permite descender a la profundidad de la gran razón del cuerpo, una razón desarrollada «sobre una base sensualista, sobre los prejuicios de los sentidos, es decir creyendo en la verdad de los juicios de los sentidos» ${ }^{31}$.

La conciencia y la razón se convierten en aspectos particulares de la vida del cuerpo, que, a su vez, es considerado como un conjunto jerárquico de instintos y pulsiones, de procesos organizados y coordinados, excepto en el caso de la enfermedad y la decadencia. Los instintos son procesos susceptibles de cambio, están estrechamente ligados a las evaluaciones y expresan el trabajo de ordenación selectiva de la realidad dictada por dichas evaluaciones como preferencias fundamentales ${ }^{32}$. Teniendo en cuenta esta retroalimentación, se puede afirmar que el cuerpo débil creó el cristianismo, pero también, y al mismo tiempo, que el cristianismo perpetúa el cuerpo débil, porque llega un momento en que los gustos y los juicios son incorporados y pasan a convertirse en instintos que nacen espontáneamente sin necesidad de estímulo. Por ello, Nietzsche anima a estudiar la moral como causa, pero también al mismo tiempo, como consecuencia y como síntoma ${ }^{33}$. Es posible lanzar la pregunta sobre qué nos enseña un determinado punto de vista sobre quién lo hace. Instintos y pulsiones son tanto el origen de los valores como el producto de su incorporación o encarnación (Einverleibung), de modo que no hemos de pensarlos como principio absoluto o punto de partida, sino como procesos dinámicos con posibili-

28 CI «La razón en la filosofía».

29 La genealogía de la moral III $\$ 12$ (= GM).

30 Fornari, Maria Cristina (2013), «Para una fisiología de la genealogía», Estudios Nietzsche, n 13, pp. $27-37$.

31 FP 1887 9[63], vol. IV p. 252.

32 Cf. Wotling, Patrick (2016), “Oui, l'homme fut un essai” La philosophie de l'avenir selon Nietzsche. Paris: PUF.

33 GM «Prólogo» 6. 
dad de cambio. Cada "instinto" es instinto hacia "algo bueno", visto desde algún punto de vista, y ha sido cultivado como condición de existencia temporal ${ }^{34}$. Un instinto es el efecto ulterior de una estimación de valor que ha sido conservada y fomentada durante mucho tiempo. Del mismo modo, la percepción (Wahrnehmen) es esencialmente un tener por verdadero (als-wahr-nehmen), pues todas las percepciones sensoriales están impregnadas de juicios de valor.

Una moral es una interpretación ligada a un sistema de valores que expresa las condiciones de vida de un tipo humano particular y sus estimaciones de valor, que siguen «las leyes del perspectivismo, en cada caso según cómo sea un ser humano o un pueblo - lo que está cerca, es importante, necesario, etc.» ${ }^{35}$. Es el producto de la elaboración de la realidad efectuada por el cuerpo y sus procesos constitutivos, una semiótica de los afec$\operatorname{tos}^{36}$. El cuerpo es fuente de interpretaciones, que, a su vez, atestiguan el estado del cuerpo que interpreta, un cuerpo que no es ajeno a la voluntad, al dolor y al tiempo. Y pretender eliminar la voluntad y dejar en suspenso la totalidad de los afectos sería equivalente a castrar el intelecto ${ }^{37}$, incluso incapacitarlo, pues únicamente puede funcionar desde sus propias formas perspectivistas ${ }^{38}$.

Este vínculo entre cuerpo e interpretación explica la apreciación de las doctrinas de pensamiento en términos de salud y enfermedad. Según Nietzsche, la filosofía ha de ser comprendida como una forma de tratamiento o curación (Heil, Heilung) de la cultura ${ }^{39}$. El médico filósofo elabora un diagnóstico, porque hay cosas como la enfermedad que no se refutan, sino que han de ser curadas ${ }^{40}$. Para obtener resultados culturales, hay que comenzar por convencer al cuerpo ${ }^{41}$, y la fuerza de los conocimientos no reside en su grado de verdad, sino en su incorporabilidad (Einverleibtheit) ${ }^{42}$, en su carácter de condición para la vida ${ }^{43}$. Por ello, el médico-filósofo entiende la moral como sintomatología, como un «hablar por signos» (Zeichenrede) ${ }^{44}$.

La genealogía es una historia de la génesis del pensamiento (Entstehungsgeschichte des Denkens), es conocimiento de los procesos de creación y conservación de las distintas culturas. Nos permite cuestionar el valor de los valores, de las ideas, de las prácticas y las instituciones que son mantenidas en el presente, que en muchos casos legitiman un marco social coercitivo. Elimina el carácter de dogma de todo conocimiento, de verdad necesaria,

34 FP 1884 26[72], vol. III p. 552.

35 FP 1884 25[460], vol. III p. 532-533.

36 MBM $\$ 187$

37 GM III $1 \S 2$

38 GC $\$ 130$ y $\$ 274$

39 Faustino, Marta (2017), «Nietzsche's Therapy of Therapy», Nietzsche-Studien, n 46, pp. 82-104, p. 92.

40 Conill, Jesús (2017), «Crítica genealógica de la cultura política moderna y sus implicaciones para el futuro de la democracia, a partir de Humano, demasiado humano», en: Céline Denat y Patrick Wotling (Dir.), Humain, trop humain et les débuts de la réforme de la philosophie. Reims: Éditions et Presses Universitaires de Reims, pp. 197-216, p. 202.

41 CI «Incursiones de un intempestivo» $\$ 47$

42 Cf. Lemm, Vanessa (2015), «Verdad, incorporación y probidad en Nietzsche», Estudios Nietzsche, $\mathrm{n}^{\circ}$ 15, pp. 63-81.

$43 \mathrm{GC} \$ 110$.

44 CI «Los "mejoradores" de la humanidad» $\$ 1$ 
y pone de relieve lo contingente y lo histórico. Así pues, crea un conocimiento con potencial de efectividad práctica para el presente; sabiendo que la comunicación y la discusión de argumentos es posible, «emprende el genealogista la deconstrucción histórica de un determinado valor que legitima en el presente el poder de los grupos hegemónicos» ${ }^{45}$.

Nietzsche critica el anhelo de sostén de quien busca el conocimiento "en sî" o una fe que haga innecesaria la creación de valores. La voluntad de verdad es síntoma de una impotencia para crear $^{46}$. Un presupuesto de la vida es que algo tiene que ser tenido por verdadero, pero no que lo sea. Así, el mundo verdadero se convierte en una fábula ${ }^{47}$ que cumple unas funciones pragmáticas. La valoración de "lo verdadero" expresa unas condiciones de conservación y crecimiento de un tipo de vida, pero no la verdad ${ }^{48}$. Hemos proyectado nuestras condiciones de conservación como si fueran predicados del ser ${ }^{49}$. Por tanto, Nietzsche critica, ante todo, el estatuto ontológico de la verdad y de la moral, y su posición privilegiada por encima del punto de vista del valor, «el punto de vista de las condiciones de conservación y de aumento (...) "valor" es esencialmente el punto de vista para el aumento o la disminución de estos centros de dominio» ${ }^{50}$.

En su lugar, reivindica el conocimiento al servicio de la vida. De ahí que reflexione sobre el valor de la historia desde la perspectiva de la cultura, y redefina el sentido histórico como «la capacidad de adivinar con rapidez la jerarquía de las valoraciones según las cuales han vivido un pueblo, una sociedad, un ser humano» ${ }^{51}$. La historia es un gran laboratorio axiológico, pero el perspectivismo de Nietzsche es crítico y no cae en relativismo. Tal pensamiento indeciso nos forzaría a renunciar a cualquier potencial de conocimiento con sentido transformador, y perpetuaría precisamente el pensamiento nihilista y ontologizante que Nietzsche critica ${ }^{52}$. Tras el reconocimiento de la perspectiva, la búsqueda se transvalora. Esta es una exigencia planteada explícitamente: la genealogía aporta conocimiento sobre las condiciones y circunstancias en que surgen, se desarrollan y se modifican los valores, pero la tarea del filósofo no es meramente descriptiva, sino que consiste en solucionar el problema del valor, en determinar la jerarquía de los valores ${ }^{53}$.

Recientemente, Langsam defendía el carácter subjetivista de los valores en Nietzsche ${ }^{54}$. En efecto, los valores son subjetivos en la medida en que son acuñados por las personas y no existen en sí mismos, y tampoco poseen un valor independiente del tipo de seres

45 Romero Cuevas, José Manuel (2015), «Perspectivismo y crítica social. De Nietzsche a la Teoría Crítica», Logos. Anales del Seminario de Metafísica, $\mathrm{n}^{\circ}$ 48, pp. 141-163, p. 151.

46 Cf. FP 1887 9[60], vol. IV p. 249: «La creencia de que el mundo que debería ser es, existe realmente, es una creencia de los improductivos que no quieren crear un mundo como debe ser»

47 CI «Cómo el mundo verdadero acabó convirtiéndose en fábula»

48 Conill, Jesús (2011), «Introduzione. Hermenéutica tropológica de la verdad en Nietzsche», en: Pietro Gori y Paolo Stellino (Eds.), Teorie e pratiche della verità in Nietzsche, Pisa: Edizioni ETS, pp. 13-24, p. 15.

49 FP 1887 9[38], vol. IV pp. 242-243.

50 FP 1887 11[73], vol. IV p. 388.

51 MBM $\$ 224$.

52 Navratil, Michael (2017), «,Einige Sprossen zurück“. Metaphysikkritik, Perspektivismus und die Gültigkeit der Perspektiven in Nietzsches Menschliches, Allzumenschliches», Nietzsche-Studien, $\mathrm{n}^{\circ}$ 46, pp. 58-81, p. 17.

53 GM «Prólogo» §6 y GM I §17, «Nota».

54 Langsam, Harold (2018), «Nietzsche and value creation: subjectivism, self-expression, and strength», Inquiry, vol. $61, \mathrm{n}^{\circ} 1$, pp. 100-113. 
humanos que producen. El anti-realismo generalizado de Nietzsche concierne también al modo de existencia de los valores, que no existen en una realidad independiente, sino que son creados como resultado de la respuesta afectiva de los seres humanos frente al mundo ${ }^{55}$. No obstante, a mi juicio, la clave no reside en la oposición "objetivo o subjetivo", ni tampoco "absoluto o relativo", sino, de nuevo, en el resultado que dicho valor produce, en sus consecuencias respecto a las formas de sociedad y la elevación de la cultura. El perspectivismo nos enseña que los valores poseen un valor situado, que depende del fin último ( ¿ivalioso para qué?» ${ }^{56}$ ) y del contexto vital que les otorga sentido, pero no deriva en subjetivismo. Lanzar la pregunta por el valor de los valores - que, recordemos, es el objeto de La genealogía de la moral-, presupone reconocer que no todos los valores valen lo mismo. Por ello, la tarea (Aufgabe) que el filósofo debe solucionar es establecer las jerarquías del valor, determinar el orden de rango (Rangordnung ${ }^{57}$. Además, no hemos de olvidar que el proyecto nietzscheano no es meramente individualista, sino que tiene una dimensión colectiva y relacional muy importante, en lo que se refiere a la reforma de la cultura y la constante preocupación por la formación de unos tipos de seres humanos más elevados, y también posee una dimensión política, que se aprecia especialmente en El Anticristo ${ }^{58}$, el libro de la transvaloración de los valores.

Por lo que se refiere a la noción de la objetividad, esta noción es, a su vez, víctima de una transvaloración y pasa a significar la multiplicación de las perspectivas. A partir de dicho cúmulo de perspectivas, se puede elaborar un conocimiento con pretensión de objetividad, pero despojado de cualquier aparato metafísico que le confiriese una autoridad extramoral.

Ver alguna vez las cosas de otro modo, querer verlas de otro modo, es una no pequeña disciplina y preparación del intelecto para su futura objetividad, - entendida esta última no como «contemplación desinteresada» (que, como tal, es un noconcepto y un contrasentido), sino como la facultad de tener nuestro pro y nuestro contra sujetos a nuestro dominio y de poder separarlos y juntarlos: de modo que sepamos utilizar en provecho del conocimiento cabalmente la diversidad de las perspectivas y de las interpretaciones nacidas de los afectos. A partir de ahora, señores filósofos, guardémonos mejor, por tanto, de la peligrosa y vieja patraña conceptual que ha creado un «sujeto puro del conocimiento, sujeto ajeno a la voluntad, al dolor, al tiempo», guardémonos los tentáculos de conceptos contradictorios tales como «razón pura», «espiritualidad absoluta», «conocimiento en sí»: - aquí se nos pide siempre pensar un ojo que de ninguna manera puede ser pensado, un ojo carente en absoluto de toda orientación, en el cual debieran estar entorpecidas y ausentes las fuerzas activas e interpretativas, que son, sin embargo, las que hacen que ver sea ver-algo, aquí se nos pide siempre, por tanto, un contrasentido y un no-concepto de ojo. Existe únicamente un ver «perspectivista», únicamente un

55 Rutherford, Donald (2018), «Nietzsche as perfectionist». Inquiry, vol. 61, n 1 , pp. 42-61.

56 GM I $\$ 17$

57 Ibid.

58 Souladié, Yannick (2011), «L'Antichrist en tant que personnage politique», en: Céline Denat y Chiara Piazzesi (eds.), Nietzsche penseur de la politique? Nietzsche, penseur du social? Pisa: ETS, pp. 187-200. 
«conocer» perspectivista; y cuanto mayor sea el número de ojos, de ojos distintos que sepamos emplear para ver una misma cosa, tanto más completo será nuestro concepto de ella, tanto más completa será nuestra objetividad ${ }^{59}$.

Este pasaje representa una genuina defensa de un tipo de conocimiento que se sabe perspectivista, que ha sido creado gracias a varios agentes que observan, conscientes de sus intereses, y que ponen en primera línea la cuestión de valor. Si bien el ángulo desde el que observan cada uno de dichos ojos es limitado, ya que todos ellos están situados en un punto particular del espacio y tiempo, está dentro de su poder elaborar una interpretación con pretensión de veracidad. De acuerdo con Gori y Stellino, el perspectivismo se basa en un modelo relacional, según el cual la validez de cada perspectiva específica ha de ser evaluada conforme a la relación que mantiene con otras perspectivas ${ }^{60}$. Esta llamada a la multiplicación de las perspectivas constituye una invitación al experimentalismo óptico, a apreciar la riqueza y las ambigüedades del mundo no sólo con el mayor número de ojos posible, sino también con ojos cualitativamente distintos.

Pero esta "nueva objetividad" no es un fin en sí mismo, sino que sienta las bases para después juzgar si determinados juicios enriquecen o empobrecen nuestra experiencia vital. Las valoraciones surgen de centros de interpretación diversos que, al mismo tiempo, construyen nuestro horizonte variable de perspectivas. Medimos el mundo con arreglo a nuestro horizonte, entendido como el ámbito de visión que abarca todo lo que es accesible a partir de una determinada situación, de modo que una nueva objetividad, una multiplicación de las perspectivas, nos permitirá interpretar en un todo mayor y ganar libertad ${ }^{61}$.

\section{La perspectiva de la "cría" como fisiología de la moral}

A lo largo del artículo, hemos analizado cómo Nietzsche reinterpreta las concepciones tradicionales del conocimiento en la teoría perspectivista de los afectos, e invierte la comprensión tradicional de la epistemología al sostener que la verdad no tiene un valor en sí misma, sino que su valor depende si acaso dicho "tener-por-verdadero" potencia la vida. Este criterio es asimismo perspectivista, porque la elevación de la vida es un resultado dependiente del sujeto, de la situación y de múltiples variables de la acción, lo cual impide especificar parámetros universales. Además, permite la proliferación de interpretaciones desde la óptica de la vida, al mismo tiempo que se mantiene un principio regulador que permite distinguir entre mejores y peores interpretaciones en contextos concretos ${ }^{62}$. Nietzsche era un ávido lector de su tiempo, y sus lecturas de lingüística comparada, estudios sobre las religiones y de etnología, le llevan a desarrollar una perspectiva comparativa que Figl denomina "hermenéutica transcultural"63,

59 GM III $\$ 12$

60 Gori, Pietro y Stellino, Paolo (2018), «Moral Relativism and Perspectival Values», op. cit., p. 163.

61 A $\$ 117$

62 Cf. Schrift, Alan D. (1987), «Between Perspectivism and Philology: Genealogy as Hermeneutic», NietzscheStudien, $\mathrm{n}^{\circ} 16,91-11$.

63 Figl, Johann (2014), «La hermenéutica transcultural: La interpretación nietzscheana de las religiones y culturas extraeuropeas», en: Jesús Conill-Sancho y Diego Sánchez Meca (eds.), Guía Comares de Nietzsche, Granada: Comares, pp. 123-136. 
cuyo objetivo es interpretar el dinamismo de las culturas a partir de su trasfondo valorativo. Es un privilegio el poder comparar distintas concepciones del mundo, pero «nuestra fuerza tiene que ponerse de manifiesto en el modo en que elegimos; tenemos que ser jueces ${ }^{64}$.

Ahora bien, se puede objetar que, a la hora de elucidar las diferencias de valor de los diversos valores, el "nivel de vida" es un criterio excesivamente abstracto para ser sustancial. No obstante, mi propuesta consiste en captar el discurso nietzscheano en torno a la función de los valores como instrumento de reproducción de las condiciones sociales, pues, a mi modo de ver, el pensamiento nietzscheano encuentra su máxima fecundidad en este aspecto. Gracias a la genealogía, los valores dejan de ser percibidos como realidades inmutables para ser reconocidos como instrumentos de (auto)conservación al servicio de grupos humanos concretos. A este cuestionamiento radical contribuye la pregunta sobre cómo se crían los seres humanos:

Liberado de la tiranía de los conceptos «eternos», estoy lejos, por otra parte, de precipitarme por eso en el abismo de una arbitrariedad escéptica: más bien, invito a considerar los conceptos como ensayos, con cuya ayuda determinadas especies de seres humanos se crían y a su moderación y duración $-{ }^{65}$.

Desde Humano, demasiado humano, Nietzsche elabora un análisis histórico en términos de "cría" (Züchtung). Estos análisis describen los modos en que distintos contextos históricos y culturales forman diferentes tipos de seres humanos. El conocimiento que nos proporciona la genealogía nos permite reinterpretar las transformaciones históricas como procesos de cría y problematizar cuáles son las condiciones de vida que sostienen cada forma cultural. Este uso amplio de cultura designa el conjunto de actividades humanas, las estructuras políticas y sociales, y sus productos en materia de moral, religión, arte, filosofía, etc. La cultura es la unidad de estilo de las manifestaciones de la vida de un pueblo y una época se define por el conjunto de valores que guían la vida de las personas ${ }^{66}$. ¿Pero a qué estamos aspirando con dichos valores? ¿Cuál es el valor de las costumbres y las prohibiciones?

Cría (Züchtung) y doma (Zähmung) son dos nociones que Nietzsche toma prestadas de la zoología y la botánica de su tiempo, y como tal, destacan el carácter animal del ser humano — «sólo estos termini zoológicos expresan realidades» ${ }^{67}$. La primera aparición de Züchtung ${ }^{68}$ en los textos nietzscheanos se encuentra en un fragmento póstumo de 1873, que probablemente sea un apunte de lectura del libro Physics and Politics de Walter Bagehot, economista adscrito al darwinismo:

\footnotetext{
64 FP 1877 23[85], vol. II p. 345.

65 FP 1885 35[36], vol. III p. 781.

66 Consideraciones intempestivas I, «David Strauss, escritor y apóstol» $\$ 1$

67 CI «Los mejoradores de la humanidad», §2.

68 Cabe destacar que Züchtung es un término polisémico que, si bien expresa de manera primordial una cría, puede ser traducido por variantes como «selección», «cultivo» o «disciplina» según el contexto. No obstante, a mi juicio conviene evitar traducirlo por «educación» ya que se pierde el prisma biológico. De la misma raíz son Zucht (cría, cultivo, disciplina) y Zuchtwahl (selección). Los estudiosos suelen traducir Züchtung al inglés como «breeding»y al francés como «élevage».
} 
La nacionalidad es la mayoría de las veces sólo la consecuencia de rígidas normativas de gobierno, es decir, de un tipo de cría (einer Art Züchtung) impuesta por la violencia circundante y el refregamiento, además de casarse y hablar y vivir juntos ${ }^{69}$.

Desde la perspectiva de los instintos, la moralidad de las costumbres contribuye a crear una tendencia a la obediencia y al hábito ${ }^{70}$. La dinámica de la sociedad gregaria fomenta la perspectiva compartida a partir de la cual nos es dado observar el mundo desde la costumbre, y que constituye la conciencia colectiva ${ }^{71}$. Estos procesos de cría favorecen la aparición y el mantenimiento de tipos humanos específicos, con características instintivas específicas. La cultura de un pueblo se manifiesta en la disciplina homogénea impuesta a sus instintos, y en este sentido, Salanskis habla de una «lógica de cría inconsciente ${ }^{72}$, de la cual el ejemplo de la nacionalidad es representativo. Por tanto, se ha de llevar a cabo un cuestionamiento auténtico y radical de la estructura instintiva que caracteriza al tipo humano criado por cada comunidad. Hemos de problematizar que estos procesos de condicionamiento tienen lugar tanto con nuestro consentimiento como sin él, y toda persona autónoma y soberana debería tomar las riendas de su propia cría, más allá de las lógicas del rebaño.

Sin duda, Nietzsche reconoce que los procesos de socialización responden a una necesidad de convivencia, pero advierte de los peligros de la igualación dominante y de la adaptación al punto de vista prevaleciente, tales como el estancamiento y el embrutecimiento ${ }^{73}$. Es sabido que, en La genealogía de la moral, Nietzsche sostiene que la fundación y la preservación de instituciones como la Iglesia cristiana y el Estado moderno coinciden en apuntar a la cría de un tipo específico de animal social y civilizado que es intrínsecamente confiable, predecible y consagrado al bien común. La clave de la crítica reside en poner de manifiesto que las morales universalizadoras funcionan como «medios eficaces de esclavización, al normativizar imperativamente las acciones, las motivaciones psicológicas e incluso las intenciones más íntimas» ${ }^{74}$.

Nietzsche habla de "civilización" para referirse a la emergencia de formas de organización social y política basadas en la doma del ser humano, que privilegia la autoconservación del grupo a precio de la normalización del individuo. La doma es un tipo de manipulación y debilitación de los instintos. El uso de este término es explícito si se tiene en cuenta que es el mismo proceso al que son sometidos los animales peligrosos. En este sentido ha de entenderse la expresión «bestia rubia» ${ }^{75}$, metáfora del ser humano originario previo a la civilización. La bestia rubia ha sido domada a través de la moralidad, se le ha inculcado la mala conciencia. No ha sido mejorada, sino que ha enfermado. Por otro lado, Nietzsche defiende la libera-

69 FP 1873 29[48], vol. I p. 502-503.

70 Aurora $\$ 9$.

71 Sobre perspectivismo y moral gregaria, véase la segunda parte de Gori, Pietro (2017), Nietzsche y el perspectivismo, op. cit.

72 Salanskis, Emmanuel (2016), «Un prisme de la pensée historique de Nietzsche: l'élevage», en: Bertrand Binoche y Arnaud Sorosina (eds.), Les historicités de Nietzsche, Paris: Publications de la Sorbonne, pp. 183196 , p. 185.

73 Humano, demasiado humano, $\$ 224$.

74 Sánchez Meca, Diego (2004), «Razones de la moral y exigencias de la vida: Kant contra Nietzsche», Daimon. Revista Internacional de Filosofía, $\mathrm{n}^{\circ} 33$, pp. 157-166.

75 La genealogía de la moral I §11 y El crepúsculo de los ídolos, «Los mejoradores de la humanidad», §2. 
ción de la vida animal de su condición de objeto de dominación y de explotación política ${ }^{76}$, emancipación que permitiría la pluralización de formas de vida intrínsecamente singulares.

$\mathrm{Al}$ contrario que la doma, las crías no buscan la homogenización absoluta de los seres humanos, sino el florecimiento de diferentes tipos. Por este motivo, las leyes de Manú, al distinguir entre varios tipos de seres humanos, son un ejemplo de cría, y no de doma. No obstante, no permiten la autosuperación y la elevación del ser humano ${ }^{77}$. Por ello, propongo distinguir entre dos niveles de cría cualitativamente distintos: las crías que conservan la vida y las que elevan la vida ${ }^{78}$. Esta distinción es clave, ya que encontramos pasajes en los que Nietzsche critica los procesos de cría en un modo despectivo, pero al mismo tiempo, son también numerosos los pasajes en que él mismo fantasea con la cría de seres humanos excepcionales, y piensa en «todo aquello que, con una favorable concentración e incremento de fuerzas y de tareas, podría sacarse del ser humano mediante su cría» ${ }^{79}$, de modo que existiría una diferencia cualitativa fundamental. Este segundo tipo de cría potencia el dominio como desarrollo creativo. Como vemos, frente a la habituación y la tradición, Nietzsche lanza la reflexión en torno a la posibilidad de reformar una cultura. Uno de los mecanismos de dicha reforma será la cría reflexiva de los diversos tipos de seres humanos, como proceso de elevación de sus sistemas de valores. Este tipo de cría liberadora aseguraría las condiciones necesarias para una encarnación de los valores nobles, proceso que escapa a la educación convencional cuyo alcance no es transformador, sino superficial.

\section{Conclusiones}

El perspectivismo nietzscheano surge como crítica de las corrientes positivistas y del anhelo de verdad absoluta por parte de la metafísica y la moral cristiana. Su crítica pone de relieve que el punto de vista del valor es más fundamental que el de la verdad, y que, de hecho, la verdad es un valor que se mantiene por fe. Pero la crítica genealógica descubre las condiciones experienciales de la verdad, que es transvalorada como función vital siguiendo el hilo conductor del cuerpo. El fenómeno fundamental a partir del cual surgen las interpretaciones es la experiencia vital del cuerpo. Por ello, Nietzsche nos anima a generar perspectivas forjadas en la elevación de la vida misma. Toda valoración se manifiesta como voluntad de producción de sentido mediada fisiológica, histórica y culturalmente. Así pues, Nietzsche instaura la interpretación, no como explicación, sino como figura básica del pensamiento responsable de la introducción de sentido. Ahora bien, el perspectivismo no significa en absoluto que no podamos o debamos escoger entre una

76 Lemm, Vanessa (2009), Nietzsche's Animal Philosophy: Culture, Politics, and the Animality of the Human Being, New York: Fordham University Press.

77 He analizado el caso específico de las leyes de Manú en: García-Granero, Marina (2017), «Nietzsche y las leyes de Manú en perspectiva eugenésica», en Patrici Calvo y Maria Medina-Vicent (eds.), Mirades intersubjectives en la filosofia actual, València: Societat de Filosofía del País Valencià, pp. 221-232.

78 De manera similar, en lo que respecta a la creación de valores, Dries realiza una distinción pareja entre aquellos valores que ejercen una "basic life-affirmation" y los que realizan la "Life-affirmation par excellence". Cf. Dries, Manuel (2015), «What is it like to recognize values? (The hard problem of value 2)», Nietzsche-Studien, $\mathrm{n}^{\circ} 44$, pp. 113-121.

79 MBM §203. El original alemán dice: «aus dem Menschen zu züchten wäre». 
pluralidad de puntos de vista. Ni todas las valoraciones ni todas las formas de vida tienen el mismo valor; tampoco son equivalentes en su introducción de sentido para la vida.

Finalmente, el concepto de cría en Nietzsche tiene un sentido vanguardista que pone de relieve cómo la moral, en tanto que instrumento de educación y socialización, impone una serie de interpretaciones fundamentales cuya incorporación conlleva una modificación de los instintos y de su rango en el seno de la estructura jerárquica que constituye el cuerpo. Como resultado, a largo plazo cada moral contribuye a criar unos tipos de seres humanos en detrimento de otros posibles.

Queda pendiente, no obstante, la elaboración de criterios sustanciales que expliciten cómo se eleva la vida, y ésta es la pregunta que, a mi juicio, los neonietzscheanos debemos responder en la actualidad, yendo más allá de Nietzsche, y atendiendo, no sólo a las aportaciones de otros filósofos y filósofas, sino también teniendo en cuenta las lecciones que nos proporciona la historia del siglo XX.

\section{Referencias}

Babich, Babette E. (2008), «Un problema con cuernos... el problema de la ciencia misma. La crítica de Nietzsche a la razón científica». Estudios Nietzsche, no 8, pp. 13-52.

Barrios, Manuel (1999), «Intelecto logificador y voluntad creadora en Friedrich Nietzsche», Daimon, $\mathrm{n}^{\circ} 18$, pp. 99-111.

Conill, Jesús (2011), «Introduzione. Hermenéutica tropológica de la verdad en Nietzsche», en: Pietro Gori y Paolo Stellino (Eds.), Teorie e pratiche della verità in Nietzsche, Pisa: Edizioni ETS, pp. 13-24.

Conill, Jesús (2016), «Genealogía hermenéutica de la poetización de los signos en la filosofía nietzscheana de la corporalidad», Anuario filosófico, vol. 49, no 3, pp. 609-633.

Conill, Jesús (2017), «Crítica genealógica de la cultura política moderna y sus implicaciones para el futuro de la democracia, a partir de Humano, demasiado humano», en: Céline Denat y Patrick Wotling (Dir.), Humain, trop humain et les débuts de la réforme de la philosophie. Reims: Éditions et Presses Universitaires de Reims, pp. 197-216.

Dries, Manuel (2015), «What is it like to recognize values? (The hard problem of value 2)», Nietzsche-Studien, $\mathrm{n}^{\circ} 44$, pp. 113-121.

Faustino, Marta (2017), «Nietzsche's Therapy of Therapy», Nietzsche-Studien, $\mathrm{n}^{\circ}$ 46, pp. 82-104.

Figl, Johann (2014), «La hermenéutica transcultural: La interpretación nietzscheana de las religiones y culturas extraeuropeas», en: Jesús Conill-Sancho y Diego Sánchez Meca (eds.), Guía Comares de Nietzsche, Granada: Comares, pp. 123-136.

Fornari, Maria Cristina (2013), «Para una fisiología de la genealogía», Estudios Nietzsche, $\mathrm{n}^{\mathrm{o}} 13$, pp. 27-37.

García-Granero, Marina (2017), «Nietzsche y el mejoramiento humano. Reflexiones en torno a la noción de vida», Isegoría. Revista de Filosofía Moral y Política, no 57, pp. 599-615.

García-Granero, Marina (2017), «Nietzsche y las leyes de Manú en perspectiva eugenésica», en Patrici Calvo y Maria Medina-Vicent (eds.), Mirades intersubjectives en la filosofia actual, València: Societat de Filosofía del País Valencià, pp. 221-232. 
Gori, Pietro (2017), Nietzsche y el perspectivismo, Córdoba (Argentina): Editorial Brujas.

Gori, Pietro y Stellino, Paolo (2018), «Moral Relativism and Perspectival Values», en: António Marques and João Sàágua (eds.), Essays on Value and Practical Rationality, Bern: Peter Lang, pp. 155-174.

Heit, Helmut (2016), «Naturalizing Perspectives. On the Epistemology of Nietzsche's Experimental Naturalizations», Nietzsche-Studien, $\mathrm{n}^{\circ}$ 45, pp. 56-80.

Langsam, Harold (2018), «Nietzsche and value creation: subjectivism, self-expression, and strength», Inquiry, vol. 61, $\mathrm{n}^{\circ} 1$, pp. 100-113.

Lemm, Vanessa (2009), Nietzsche's Animal Philosophy: Culture, Politics, and the Animality of the Human Being, New York: Fordham University Press.

Lemm, Vanessa (2015), «Verdad, incorporación y probidad (Redlichkeit) en Nietzsche», Estudios Nietzsche, $\mathrm{n}^{\circ} 15$, pp. 63-81.

Navratil, Michael (2017), «,Einige Sprossen zurück“. Metaphysikkritik, Perspektivismus und die Gültigkeit der Perspektiven in Nietzsches Menschliches, Allzumenschliches», Nietzsche-Studien, $\mathrm{n}^{\circ}$ 46, pp. 58-81.

Nietzsche, Friedrich (1967 ss.), Werke. Kritische Gesamtausgabe, Herausgegeben von Giorgio Colli und Mazzino Montinari. Berlin-New York: Walter de Gruyter. Edición crítica digital (eKGWB) disponible en http://www.nietzschesource.org.

Nietzsche, Friedrich (2005 ss.), Correspondencia. Madrid: Trotta, 6. vols. Edición dirigida por Luis Enrique de Santiago Guervós.

Nietzsche, Friedrich (2006 ss.), Fragmentos póstumos. Madrid: Tecnos, 4 vols. Edición dirigida por Diego Sánchez Meca.

Romero Cuevas, José Manuel (2015), «Perspectivismo y crítica social. De Nietzsche a la Teoría Crítica», Logos. Anales del Seminario de Metafísica, nº 48, pp. 141-163.

Rutherford, Donald (2018), «Nietzsche as perfectionist». Inquiry, vol. 61, n 1, pp. 42-61.

Salanskis, Emmanuel (2016), «Un prisme de la pensée historique de Nietzsche: l'élevage», en: Bertrand Binoche y Arnaud Sorosina (eds.), Les historicités de Nietzsche, Paris: Publications de la Sorbonne, pp. 183-196.

Sánchez Meca, Diego (2004), «Razones de la moral y exigencias de la vida: Kant contra Nietzsche», Daimon, no 33, pp. 157-166.

Schrift, Alan D. (1987), «Between Perspectivism and Philology: Genealogy as Hermeneutic», Nietzsche-Studien, $\mathrm{n}^{\circ}$ 16, pp. 91-11.

Souladié, Yannick (2011), «L'Antichrist en tant que personnage politique», en: Céline Denat y Chiara Piazzesi (eds.), Nietzsche penseur de la politique? Nietzsche, penseur du social? Pisa: ETS, pp. 187-200.

Wotling, Patrick (2016), “Oui, l’homme fut un essai” La philosophie de l'avenir selon Nietzsche. Paris: PUF. 